\title{
Czech Participation in International X-Ray Observatory (IXO)
}

\author{
R. Hudec, L. Pína, V. Maršíková, A. Inneman, M. Skulinová, M. Míka
}

\begin{abstract}
Here we describe the recent status of Czech participation in the IXO (International X-ray Observatory) space mission, with emphasis on the development of new technologies and test samples of X-ray mirrors with precise surfaces, based on new materials, and their applications in space. In addition, alternative X-ray optical arrangements are investigated, such as Kirkpatrick-Baez systems.
\end{abstract}

Keywords: X-ray satellites, X-ray telescopes, X-ray optics.

\section{Introduction}

The design and development of X-ray optics has a long tradition in the Czech Republic (e.g. Hudec et al., 1991, 1999, 2000, 2001, Inneman et al. 1999, 2000). A range of various related technologies have been exploited and investigated, including technologies for future large, light-weight X-ray telescopes.

Future large space X-ray telescopes (such as IXO considered by ESA or IXO/Constellation $\mathrm{X}$ by NASA) require precise, light-weight X-ray optics based on numerous thin reflecting shells. Novel approaches and advanced technologies need to be developed and exploited. In this paper, we refer to Czech efforts in connection with IXO (now Athena), focusing on the results of test X-ray mirror shells produced by glass thermal forming (GTF) and by shaping $\mathrm{Si}$ wafers. Both glass foils and Si wafers are commercially available, have excellent surface microroughness of a few $0.1 \mathrm{~nm}$, and low weight (the volume density is $2.5 \mathrm{~g} \cdot \mathrm{cm}^{-3}$ for glass and $2.3 \mathrm{~g} \cdot \mathrm{cm}^{-3}$ for $\mathrm{Si}$ ). Technologies need to be exploited for shaping these substrates to achieve the required precise $\mathrm{X}$-ray optics geometries without degrading the fine surface microroughness.

Although glass, and more recently, silicon wafers have been considered the most promising materials for future advanced large aperture X-ray telescopes, other alternative materials are also worth further study, such as amorphous metals and glassy carbon (Marsch et al., 1997). In order to achieve sub-arsec angular resolutions, the principles of active optics need to be adopted. The International $\mathrm{X}$-ray Observatory (IXO) is a new X-ray telescope with joint participation of NASA, the European Space Agency (ESA) and the Japan Aerospace Exploration Agency (JAXA). This project supersedes both NASA's Constellation-X and ESA's XEUS mission concepts. In mid-2008, officials from ESA, NASA and JAXA headquarters agreed to conduct a joint study of IXO with a single merged set of top-level science goals. This agreement established the key science measurement requirements (White et al., 2009). The spacecraft configuration for the IXO study is a mission featuring a single large X-ray mirror, an extendible optical bench with a focal length of $\sim 20 \mathrm{~m}$ and a suite of five focal plane instruments. The X-ray instruments under study for the IXO concept include: a wide field imaging detector, a highspectral-resolution imaging spectrometer (calorimeter), a hard X-ray imaging detector, a grating spectrometer, a high timing resolution spectrometer and a polarimeter. The IXO mission concept was submitted to the U.S. Decadal Survey committee and to ESA's Cosmic Vision process.

Note added in proofs: As a consequence of US Decadal Survey, on European side (ESA) the IXO will be replaced by new project Athena. As the Athena will also use the imaging X-ray Optics similarly to IXO, the developments described in this paper refer now to Athena.

\section{Czech involvement in IXO-related studies}

At the moment, the Czech participation in IXO concentrates on: (1) participating in defining scientific goals, justification and project preparation, (2) participating in the design and development of mirror technologies. The first author of this paper was delegated as a member of the IXO Telescope Working Group. In the mirror development, we focus on supporting ESA ESTEC micropore silicon technology design and also on designing and developing alternative background technologies discussed in greater detail below.

\section{The glass foil alternative for IXO}

Glass science and technology has a long tradition in the Czech Republic. At the same time, glass technol- 
ogy is one of most promising technologies for producing mirrors for IXO, as the volume density of glass is nearly four times less than the volume density of electroformed nickel layers. Glass foils can be used as flats or may be shaped or thermally slumped to achieve the required geometry. Thermal forming of glass is not a new technology, and it has been used in various sectors of the glass industry and in glass art, as well as in the production of Cherenkov mirrors. However, the application of this technology in X-ray optics is related with the need to improve accuracy significantly and minimize errors. As the first step, small (various sizes typically less than $100 \times 100 \mathrm{~mm}$ ) glass samples of various types provided by various manufacturers were used and thermally shaped. The geometry was either flat or curved (cylindrical or parabolic). The project continued with larger samples (up to $300 \times 300 \mathrm{~mm}$ ) and further profiles. Recent efforts have focused on optimizing the relevant parameters of both glass material and substrates, as well as the parameters of the slumping process.

Various approaches have been investigated (Figure 1). We note that these are not quite identical with efforts by another teams (e.g. Zhang et al., 2010, Ghigo et al., 2010). The glass samples were thermally formed at Rigaku, Prague, and also at the Institute of Chemical Technology in Prague. For large samples $(300 \times 300 \mathrm{~mm})$, facilities at the Optical Development Workshop in Turnov were used. The strategy is to develop a technology suitable for inexpensive mass production of thin X-ray optics shells, i.e., to avoid expensive mandrels and techniques that are not suitable for mass production or that are too expensive. Numerous glass samples have been shaped and tested in order to find out the optimal parameters. The shapes and profiles of both mandrels, as well as the resulting glass replicas, have been carefully measured using metrological devices. The results show that the quality of the thermal glass replica can be significantly improved by optimizing the material and improving the design of the mandrel, by modifying the thermal forming process, as well as by optimizing the temperature (Figure 2). After the modifications and improvements, some of them significant, we obtained the resulting deviation of the thermally formed glass foil from the ideal designed profile less than $1 \mu \mathrm{m}$ (peak to valley value) in the best case. This value is however strongly dependent on the exact temperature, so we believe that further improvements are still possible.

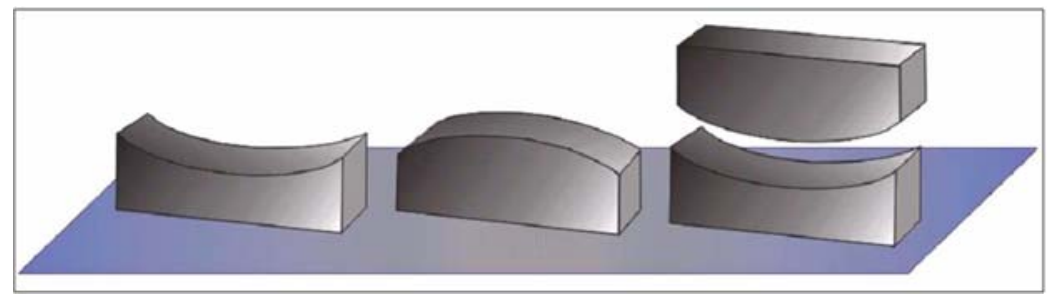

Fig. 1: The three investigated glass thermal arrangements: convex and concave mandrels and a double mandrel

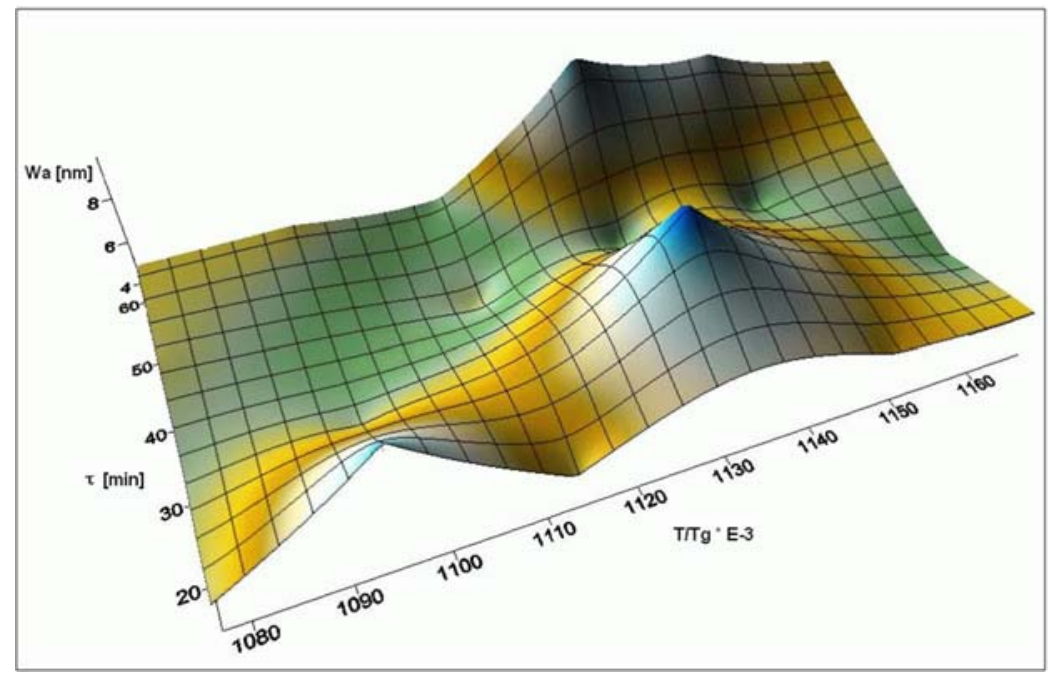

Fig. 2: An example of the optimization studies performed for glass thermal forming: optimization map for waviness (waviness Wa as function of forming process parameters i.e. duration of the thermal forming process $(\tau)$ and temperature $(T), T_{g}$ means glass transformation temperature) 
The fine original microroughness (typically better than $1 \mathrm{~nm}$ ) of the original float glass foil was found not to be degraded by the thermal forming process. We note that our approach in thermal glass forming is different from the approaches used by other authors. Recent efforts have been devoted to optimizing the whole process, using and comparing different forming strategies etc., as the final goal is to further improve the forming accuracy to less than $0.1 \mu \mathrm{m}$ values. For the near future, we plan to continue these efforts together with investigations of computer-controlled forming of glass foils (according to the principles of active optics).

\section{The silicon wafer alternative}

Silicon is a relatively light material and already during the manufacturing process it is lapped and polished (either on one side or on both sides) to very fine smoothness (better than a few $0.1 \mathrm{~nm}$ ) and thickness homogeneity (of the order of $1 \mu \mathrm{m}$ ). Another obvious alternative, recently considered as one of most promising for high-precision X-ray optics for IXO, is the use of X-ray optics based on commercially available silicon wafers manufactured mainly for the purposes of the semiconductor industry.

The main advantages of the application of $\mathrm{Si}$ wafers in space X-ray optics are (i) the volume density, which is more than 4 times lower than the electroformed nickel used in the past for galvanoplastic replication of multiply nested X-ray mirrors, and slightly less than the alternative approach of glass foils, (ii) very high thickness homogeneity, typically less than $1 \mu \mathrm{m}$ over $100 \mathrm{~mm}$, and (iii) very small surface microroughness either on one side or on both sides (typically of the order of a few $0.1 \mathrm{~nm}$ or even less, e.g. Figure 3). Silicon wafers were expected to be used in the ESA XEUS project and are still under consideration for the IXO (now Athena) project. The recent baseline optics for the IXO X-ray telescope design is based on X-Ray High Precision Pore Optics (X-HPO), a technology currently under development with ESA funding (RD-Opt, RD-HPO), in view of achieving large effective areas with low mass, reduced telescope length, high stiffness, and a monolithic structure, favoured for handling the thermal environment and for simplifying the alignment process (Bavdaz et al. 2010). In addition, due to the higher packing density and the associated shorter mirrors required, the conical approximation to the Wolter-I geometry becomes possible. The X-HPO optics is based on ribbed $\mathrm{Si}$ wafers stacked together. The Si wafers to achieve the conical approximation are formed by stacking large number of plates to- gether using a mandrel. The typical size of the $\mathrm{Si}$ wafers is $10 \times 10 \mathrm{~cm}$.

There are also alternative X-ray optics arrangements with the use of $\mathrm{Si}$ wafers. In this paper, we refer to the development of an alternative design of innovative precise X-ray optics based on Si wafers. Our approach is based on two steps, namely (i) developing dedicated $\mathrm{Si}$ wafers with properties optimized for use in space X-ray telescopes and (ii) precisely shaping the wafers to the optical surfaces (Figure 4). Stacking to achieve nested arrays is performed after the wafers have been shaped. In this approach, Multi Foil Optics (MFO) is thus created from shaped Si wafers (Figure 5). For more details on MFO, see Hudec et al. (2005).

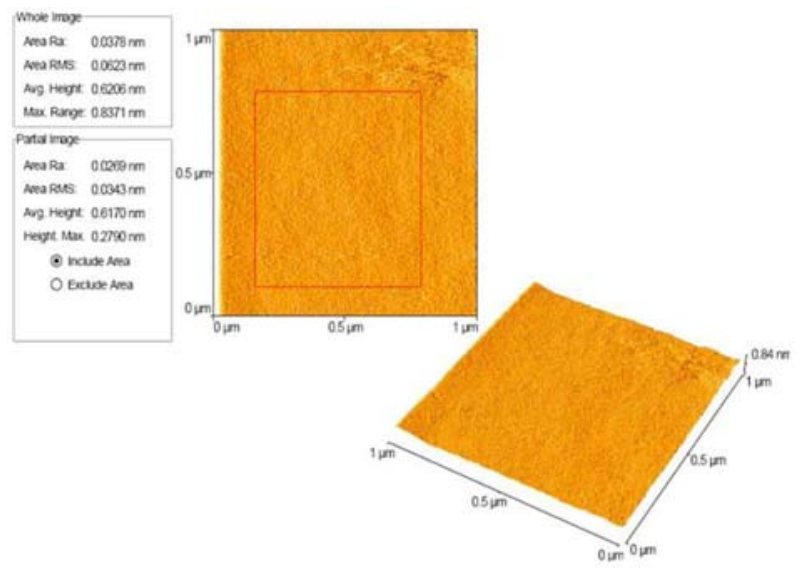

Fig. 3: AFM mesurement results for Si wafers

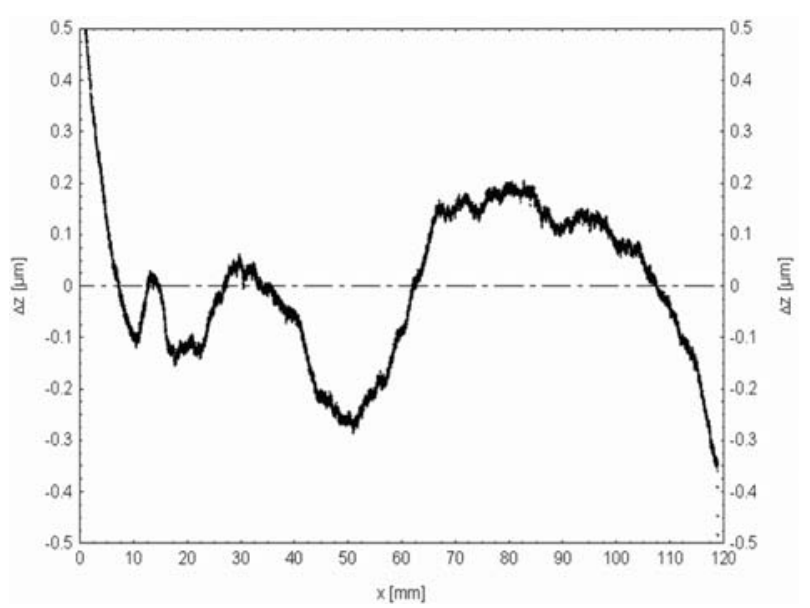

Fig. 4: Taylor-Hobson profilometric measurement of a bent $\mathrm{Si}$ wafer

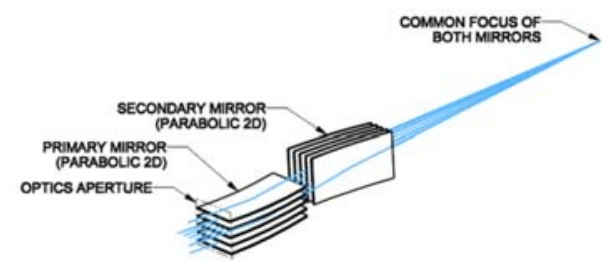

Fig. 5: Multi Foil Optics (MFO) in the Kirkpatrick-Baez (K-B) arrangement 
This alternative approach does not require the $\mathrm{Si}$ wafers to have a ribbed surface, so problems with transferring any deviation, stress, and/or inaccuracy from one wafer to the neighbouring plates or even to the whole stacked assembly will be avoided. However, suitable technologies for precise stacking of optically formed wafers to a multiple array have to be developed.

The Si wafers available on the market are designed for use mainly in the semiconductor industry. It is obvious that the requirements of this industry are not the same as the requirements of precise space X-ray optics. Si wafers are a monocrystal (single crystal) with some specifics, and this must also be taken into account. Moreover, Si wafers are fragile, and it is very difficult to bend and/or shape them precisely (for thicknesses required for X-ray telescopes, i.e. around $0.3-1.0 \mathrm{~mm}$. An exception is thin $\mathrm{Si}$ wafers below $0.1 \mathrm{~mm}$ in thickness. However, these can be hardly used in this type of X-ray optics because of diffraction limits. Also, while their thickness homogeneity is mostly perfect, the same is not true for commercially available wafers for their flatness (note that we refer here to the deviation of the upper surface of a free-standing Si wafer from an ideal plane, while in the semiconductor community flatness is usually represented by a set of parameters).

In order the achieve the very high accuracy required by future large space X-ray telescopes like ESA/NASA/JAXA IXO, now Athena by ESA, the parameters of the $\mathrm{Si}$ wafers need to be optimized (for application in X-ray optics) at the production stage. For this purpose we have established and developed a multidisciplinary working group including specialists from the development department of the $\mathrm{Si}$ wafer industry with the goal to design and manufacture Si wafers with improved parameters (mostly flatness) optimized for application in X-ray telescopes. It should be noted that the manufacture of silicon wafers is a complicated process with numerous technological steps and with many free parameters that can be modified and optimized to achieve optimal performance. This can also be useful for further improving the quality of X-HPO optics. As we are dealing with high-quality X-ray imaging, the smoothness of the reflecting surface is important. The standard microroughness of commercially available Si wafers (we have used the products of ON Semiconductor, Czech Republic) is of the order of $0.1 \mathrm{~nm}$, as confirmed by several independent measurements by various techniques including the Atomic Force Microscope (AFM). This is related to the method of chemical polishing used in the manufacture of Si wafers. The microroughness of Si wafers exceeds the microroughness of glass foils and most other alternative mirror materials and substrates. The flatness (in the sense of the deviation of the upper surface of a free- standing Si wafer from a plane) of commercially available Si wafers was however found not to be optimal for use in high-quality (order of arcsec angular resolutions) X-ray optics. Most Si wafers show deviations from the plane of the order of a few tens of microns. After modifying the technological process during $\mathrm{Si}$ wafer manufacture, we were able to reduce this value to just a few microns. Also, the thickness homogeneity was improved. In collaboration with the manufacturer, further steps are planned to improve the flatness (deviation from an ideal plane) and the thickness homogeneity of Si wafers. These and planned improvements introduced at the Si wafer manufacture stage can also be applied for other designs of $\mathrm{Si}$ wafer optics including X-HPO, and can play a crucial role in the IXO project.

The X-ray optics design for IXO (now Athena) is based on the Wolter 1 arrangement, and hence requires curved surfaces. However, due to the material properties of monocrystalline $\mathrm{Si}$, Si wafers (except very thin ones) are extremely difficult to shape. It is obvious that we have to overcome this problem in order to achieve the fine accuracy and stability required for future large X-ray telescopes. The final goal is to provide optically shaped $\mathrm{Si}$ wafers with no or little internal stress. Three different alternative technologies for shaping $\mathrm{Si}$ wafers have been designed and tested to achieve precise optical surfaces. The samples shaped and tested were typically 100 to $150 \mathrm{~mm}$ large, typically 0.6 to $1.3 \mathrm{~mm}$ thick, and were bent to either cylindrical or parabolic test surfaces.

The development described here is based on a scientific approach, and hence the large number of samples formed with different parameters must be precisely measured and investigated in detail. Especially precise metrology and measurements play a crucial role in this type of experiment. The samples of bent wafers with the investigated technologies have been measured, including Taylor-Hobson mechanical and STILL optical profilometry, as well as optical interferometry (ZYGO) and AFM (Atomic Force Miscroscope) analyses (Figure 3). It has been confirmed that all these three technologies do not degrade the intrinsic fine microroughness of the wafer. While the two physical/chemical technologies exploited give peak-to-valley (PV) deviations (of the real surface of the sample compared with the ideal optical surface) of less than 1 to $2 \mu \mathrm{m}$ over the $150 \mathrm{~mm}$ sample length, as preliminary values, the deviations of the first thermally bent sample are larger, of the order of $10 \mu \mathrm{m}$. Taking into account that the applied temperatures, as well as other parameters, were not optimized for this first sample, we anticipate that the PV (peak to valley) value can be further reduced down to the order of $1 \mu \mathrm{m}$ and perhaps even below. Fine adjustments of the parameters can also further improve the accuracy of the results for the other two techniques. 


\section{$5 \quad \mathrm{~K}-\mathrm{B}$ alternative for IXO}

Although the Wolter systems are generally well known, Hans Wolter was not the first to propose Xray imaging systems based on reflection of X-rays. In fact, the first grazing incidence system to form a real image was proposed by Kirkpatrick and Baez in 1948. This system consists of a set of two orthogonal parabolas of translation. The first reflection focuses to a line, which is focused by the second surface to a point. This was necessary to avoid the extreme astigmatism suffered from a single mirror, but it still was not free of geometric aberrations. Nevertheless, the system is attractive because it is easy to construct the reflecting surfaces. These surfaces can be produced as flat plates and then mechanically bent to the required curvature. In order to increase the aperture a number of mirrors can be nested together, but it should be noted that this nesting introduces additional aberrations. This configuration is used mostly in experiments not requiring a large collecting area (solar, laboratory). Recently, however, large modules of KB mirrors have also been suggested for stellar Xray experiments.

As mentioned above, $\mathrm{Si}$ wafers are difficult to shape, especially to small radii. To overcome this difficulty, another X-ray optics arrangement can be considered, namely the Kirkpatrick-Baez (KB) system. Then the curvature radii are much larger, of the order of a few $\mathrm{km}$, while the imaging performance is similar. For the same effective area, however, the focal length of the KB system is about twice as large as the focal length of the Wolter system. Nevertheless, $\mathrm{KB}$ systems represent a promising alternative to the classical Wolter systems in future large space X-ray telescopes.

A very important factor is the ease (and hence the reduced cost) of constructing highly segmented modules based on multiply nested thin reflecting substrates in comparison with the Wolter design. While e.g. the Wolter design for IXO requires the substrates to be precisely formed with curvatures as small as $0.25 \mathrm{~m}$, the alternative $\mathrm{KB}$ arrangement uses almost flat or only slightly bent sheets. Hence the feasibility of constructing a $\mathrm{KB}$ module with the required 5 arcsec FWHM at an affordable cost is higher than for the Wolter arrangement.

Advanced KB telescopes are based on the Multi Foil Optics (MFO) approach (X-ray grazing incidence imaging optics based on numerous thin reflecting substrates/foils). The distinction between MFO and other optics using packed or nested mirrors is that MFO is based on numerous very thin (typically less than $0.1 \mathrm{~mm}$ ) substrates. The MFO KB test modules were recently designed and constructed at Rigaku Innovative Technologies Europe (RITE) in Prague, and 2 modules were tested in full aperture X-ray tests in the test facility of the University of Boulder, with preliminary results of FWHM 26 arcsec for a full stack of 24 standard Si plates at $5 \mathrm{keV}$ (Figures 6, 7).
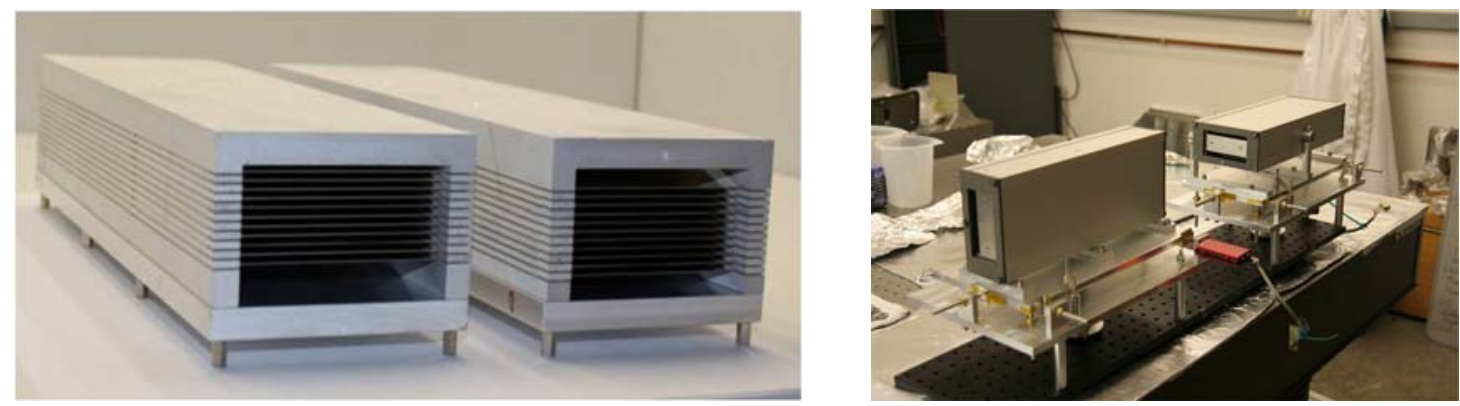

Fig. 6: Left: Test K-B modules assembled in Rigaku RITE in Prague, Right: K-B module during full aperture X-ray tests at Boulder University
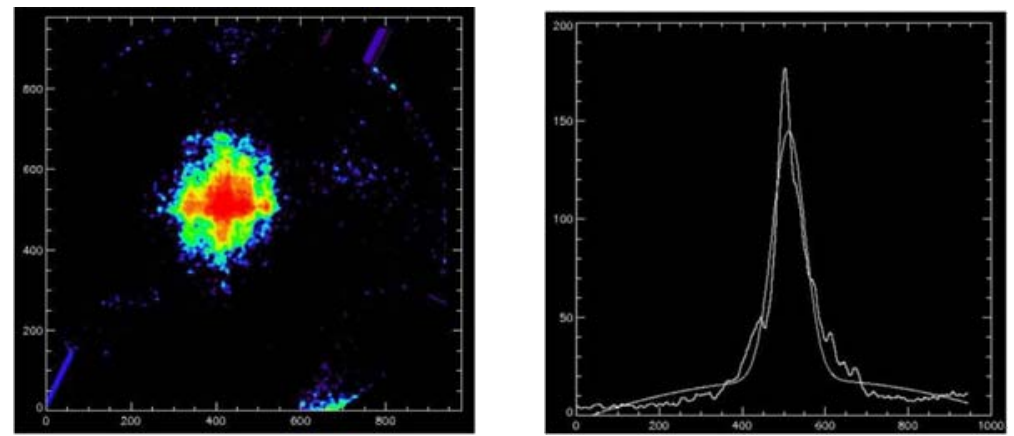

Fig. 7: The measurement results of the K-B test module with $24 \mathrm{Si}$ wafers, full aperture tests at 5 keV at University of Colorado at Boulder. The estimated FWHM is 26 arcsec 


\section{Conclusion}

Suitable technologies for future large X-ray telescopes require extensive research work. Two promising technologies suitable for future large-aperture and fine resolution X-ray telescopes, such as IXO, were exploited and investigated in detail, namely Glass Thermal Forming and $\mathrm{Si}$ wafer bending. In both cases, promising results have been achieved, with peak-tovalley deviations of the final profiles from the ideal profiles being of the order of $1 \mu \mathrm{m}$ in the best cases, with space for further essential improvements and optimization. In the Czech Republic, an interdisciplinary team with 10 members is cooperating closely with experienced specialists, including researchers from a large company producing Si wafers. Si wafers have been successfully bent to the desired geometry by three different techniques. In the best cases, the accuracy achieved for a $150 \mathrm{~mm}$ Si wafer is $1-2 \mu \mathrm{m}$ for deviation from the ideal optical surface. Experiments are continuing in an attempt to further improve the forming accuracy.

\section{Acknowledgement}

We acknowledge the support provided by the Grant Agency of the Academy of Sciences of the Czech Republic, grant IAAX 01220701, by the Ministry of Education and Youth of the Czech Republic, projects ME918, ME09028 and ME09004. The investigations related to the ESA IXO project were supported by ESA PECS Project No. 98038. M.S. acknowledges support from a junior grant from the Grant Agency of the Czech Republic, grant 202/07/P510. We also acknowledge collaboration with Drs. J. Sik and M. Lorenc from ON Semiconductor Czech Republic, and with the team of Prof. Webster Cash from University of Colorado at Boulder for X-ray tests of $\mathrm{KB}$ modules in their X-ray facility.

\section{References}

[1] Hudec, R., Valníček, B., Červencl, J., et al.: SPIE, 1991, 1 343, 162.

[2] Hudec, R., Pína, L., Inneman, A.: SPIE, 1999, 3 766, 62 .

[3] Hudec, R., Pína, L., Inneman, A.: SPIE, 2000, $4012,422$.

[4] Hudec, R., Inneman, A., Pína, L.: Lobster-Eye: Novel X-ray Telescopes for the 21st Century, New Century of $X$-ray Astronomy, ASP Conf. Proc., 2001, 251, 542.

[5] Hudec, R., Pína, L., Inneman, A., et al.: SPIE, 2005, 5 900, 276 .
[6] Inneman, A., Hudec, R., Pína, L., Gorenstein, P.: SPIE, 1999, $\mathbf{3} \mathbf{7 6 6}, 72$.

[7] Inneman, A., Hudec, R., Pína, L.: SPIE, 2000, 4 138, 94.

[8] Kirkpatrick, P., Baez, A. V.: J. Opt. Soc. Am. 38, 766 (1948).

[9] Marsch, H., et al.: Introduction to Carbon Technologies, University of Alicante, 1997.

[10] White, N. E., Hornschemeier, A. E.: Bulletin of the American Astronomical Society, 2009, Vol. 41, p. 388.

[11] White, N. E., Parmar, A., Kunieda, H., International X-ray Observatory Team, 2009, Bulletin of the American Astronomical Society, Vol. 41, p. 357 .

[12] http://ixo.gsfc.nasa.gov

[13] Bavdaz, M., et al.: Proceedings of the SPIE, 2010, Vol. 7 732, pp. 77321E-77321E-9.

[14] Zhang, W. W., et al.: Proceedings of the SPIE, 2010, Vol. 7 732, pp. 77321G-77321G-8.

[15] Ghigo, M., et al.: Proceedings of the SPIE, 2010, Vol. 7 732, pp. 77320C-77320C-12.

René Hudec

E-mail: r.hudec@asu.cas.cz

Astronomical Institute

Academy of Sciences of the Czech Republic

CZ-25165 Ondřejov, Czech Republic

Czech Technical University in Prague

Faculty of Electrical Engineering

Technická 2, CZ-166 27 Prague, Czech Republic

Ladislav Pína

Czech Technical University in Prague

Faculty of Nuclear Engineering

Břehová 78/7, CZ-110 00 Prague, Czech Republic

Veronika Maršíková

Adolf Inneman

Rigaku Innovative Technologies Europe, s. r. o.

Novodvorská 994, CZ-142 21 Prague 4,

Czech Republic

Michaela Skulinová

Astronomical Institute

Academy of Sciences of the Czech Republic

CZ-251 65 Ondřejov, Czech Republic

Martin Míka

Institute of Chemical Technology

Technická 5, CZ-166 28 Prague, Czech Republic 